\title{
Insight into the pathomorphology of the distal border of the equine navicular bone
}

\author{
Martyna Frątczak ${ }^{1}$, Jan Włodarek ${ }^{2}$, Hieronim Frąckowiak ${ }^{1}$, Marcin Komosa $^{1}$ \\ Poznan University of Life Sciences, ${ }^{1}$ Faculty of Veterinary Medicine and Animal Sciences, \\ Department of Animal Anatomy, ${ }^{2}$ Faculty of Veterinary Medicine and Animal Sciences, \\ Institute of Veterinary Medicine, Poznan, Poland \\ Received November 14, 2016 \\ Accepted May 31, 2017
}

\begin{abstract}
Pathological changes of the equine navicular bone are found in a variety of forms in numerous old and also relatively young horses. Therefore, investigations on this small bone are of major practical significance. The current article presents a view of the alterations observed on the distal aspect of the bone and analyzes their origin and importance. As a result of pressure subjected by the deep digital flexor tendon and adaptive remodelling of the bone tissue, distal border of the navicular bone extends and becomes sharpened. Osseous distal border fragments may be a consequence of this phenomenon. However, the origin of fragments is also associated with the development of entheseophytes on the margins, which can result from stress induced by the distal impar sesamoid ligament. The authors are not unanimous about the clinical importance of fragments, but it seems that the most dangerous are large-sized ones, especially when occurring together with other lesions. An important issue is also the alterations of the nutrient foramina located on the distal border, due to the main role of this surface in blood supply. Changes in the size and shape of pathological synovial invaginations can be a sign of circulatory disturbances and abnormal turnover of the bone tissue. Some researchers link synovial invaginations to cases of lameness, but their importance is still enigmatic. Majority of studies focus on warmblood horses, and a lower interest in coldblood horses can be seen. Nonetheless, certain data are a strong argument that the breed and morphotype can affect the frequency of adverse changes.
\end{abstract}

Distal sesamoid bone, os sesamoideum distale, horse, clinical morphology, lameness

The navicular bone (os sesamoideum distale) is a boat-shaped element of the horse's distal interphalangeal joint, situated between the middle and the distal phalanx (or the short pastern and the coffin bone). The navicular bone has several surfaces that are crucial for its biomechanical functions. Dorsally, there's an articular surface lined with the hyaline cartilage for a contact with the middle phalanx. The connection with the distal phalanx is enabled by the distal border of the navicular bone which contains also a small articular surface protected by the hyaline cartilage. The flexor surface covered with the fibrocartilage tissue, which makes a smooth plane for the gliding of the deep digital flexor tendon (DDFT) is located on the palmar aspect of the bone. Both on the proximal and the distal borders of the navicular bone, nutrient foramina for blood vessels can be found. Two collateral sesamoid ligaments (CSL) keep the bone in proper position, connecting the distal end of the middle phalanx with the proximal border of the navicular bone. Additionally, the distal sesamoid impar ligament (DSIL) extends from the distal border of the navicular bone with the DDFT together to the coffin bone (Waguespack and Hanson 2010; Komosa et al. 2013b). Biomechanical analyses show that distal interphalangeal joint can move in the sagittal plane (flexion and extension movements), frontal plane (lateromedial movements), and transverse plane (rotation and sliding), each inducing stresses on the articular surfaces of the sesamoid bone and the related structures of the equine digit (Denoix 1999; Wils on and Weller 2011). The main functions of the navicular bone are to provide constant angle for the DDFT and to distribute forces acting on the distal interphalangeal joint during

Address for correspondence:

Martyna Frątczak

Department of Animal Anatomy

Poznan University of Life Sciences,

Phone: +48 737864225

Ul. Wojska Polskiego 71c, 60-625 Poznan, Poland

http://actavet.vfu.cz/ 
movement (Dyson et al. 2006). Changes of this little bone are very diverse (Plate II, Fig. 1). Most frequent pathological alterations include new bone formations (enthesophytes) on the proximal and distal borders, changes in the number and size of nutrient foramina (synovial invaginations), distal border fragments and erosions on the flexor surface (cystlike lesions) (Dyson 2008; Waugespack and Hanson 2010; Komosa et al. 2013a). In the majority of cases, the appearing bone changes are evaluated by a radiological examination but lesions are often located in soft tissues, diagnosis of which is problematic. The results obtained by different methods are not always in accordance with each other (Biggi and Dyson 2010; Claerhoudt et al. 2011). This article focuses on the distal border, which is one of the most frequently affected parts of the navicular bone. In one of the recent studies, pathological changes were found most often in the DDFT ( $91 \%$ of cases) and the distal border of the navicular bone ( $85 \%$ of cases), followed by the flexor surface of the navicular bone (78.4\%) and the navicular spongiosa (76\%) (Zani et al. 2016). In another investigation of horses with a chronic palmar foot pain, $90 \%$ of them had mild to severe lesions of the distal border (Blunden et al. 2006).

\section{Distal border fragments and enthesophytes - origin and importance}

The occurrence of distal border fragments has various causes. One hypothesis implies that fragments may be the effect of fraction of the distal border of the navicular bone, which may be linked with increased extension of the palmar cortex (Plate II, Figs 2, 3). Elongation of the distal margin can be induced by physiological or pathological turnover of the bone tissue (Dyson 2008). In short explanation, bone responses to strain in modifications, called turnover, in which osteocytes, osteoclasts and osteoblasts play main roles. In physiological conditions the process of bone resorption due to osteoclasts activity remains in equilibrium with the process of bone formation, conditioned by osteoblasts. Increased load on bone tissue may cause in disorder of this process, and as the effect, in pathological changes in micro and macroarchitecture of the navicular bone (Komosa et al. 2013b). Sandler et al. (2000) revealed the significance of increased physical activity in pathological changes of the equine limb in study, in which horses subjected to intensive exercises showed some changes within the navicular bones. Alterations included increases in the size of the vascular foramina, irregular margins at the attachment sites of the DDFT, and sclerosis of the spongiosa presented. Additionally, the number of microcracks that are one of the stimuli in bone turnover were greater in the proximal and distal ends of the bones affected. Increased thickness of the trabeculi and decreased porosity of the subchondral bone plates were also seen in this cases (Sandler et al. 2000). In the work of Bentley et al. (2007) there were presented four factors of microdamage accumulation in pathological navicular bones of older horses indicating disturbances of remodeling of this bone: low bone mass, poor osteocyte connectivity, and very low osteocyte density.

Other concepts of the origin of the distal border fragment assumes mineralization of the DSIL's insertion and fraction of formed enthesophyte due to the pressure exerted by the DDFT. Wilson et al. (2001) conducted calculation of the compressive force that the DDFT exerts on the navicular bone. The maximum DDFT force in stance in the group of horses with the navicular disease was significantly greater than in the group of normal horses. It's crucial to remember that the navicular bone is only an element of a more complex construction of equine digit and cannot be considered in isolation. Forces affecting the navicular bone impact also CSL, DSIL, DDFT and the navicular bursa (Dy son 2008).

It has been suggested that proximal and distal extensions of the navicular border may be a consequence of enthesophyte formation at insertions of the CSL and DSIL, caused by chronic stress that ligaments exert on the margins of the bone during movement. Also adhesions and lesions of the DDFT could result in such changes in the architecture 
of the navicular bone (Dyson 2008). In light of this theory, associations between occurrence of fragments and condition of adjacent ligaments and tendon could be important. Significant association between distal border fragments and the grade of DSIL was found only for the large-sized fragments in the study by Biggi and Dyson (2011). There was also a finding of large enthesophyte fractured in 4 feet; in the majority of the bones the fragments were positioned laterally and medially to the enthesophyte formation. There is a supposition that the same stress resulting in enthesophyte formation may result in a fracture. Therefore, horses with large enthesophytes may be more likely to have fractures related to stresses applied to the distal aspect of the bone. Data presented by Wright et al. (1998) are a strong argument for the hypothesis that injuries and changes in DSIL may lead to enthesophytes formation. In the study from the mentioned above author bone fragments associated with the distal border of the navicular bone were found on palmar surface, embedded in the DSIL, and their palmar surfaces were covered by fibrocartilage. On radiographs a correspondingly shape in the adjacent bone was detected. Lame horses had mineralized foci within the DSIL with organized osseous tissue and little adjacent inflammatory. Contradictory other researchers found no associations between distal aspect of the navicular bone and histological grade of the DSIL (Blunden et al. 2006; Zani et al. 2016).

Fragments occur in a variety of forms. The majority of them have an oval body, but can be also thinner and longer in shape, and no particular association between their presence and height, weight, breed, age or discipline of the horses were found (Biggi and Dyson 2011). This fractions can be seen on one part of the bone or on medial and lateral parts and unilateral or bilateral. The majority of fragments appear at the junctions between horizontal and lateral or medial sloping borders, with predominance at the lateral side (Biggi and Dyson 2011; Biggi and Dyson 2012).

The connection between the presence of fragments and navicular disease is contentious. In a radiological study by Wright (1993), no evidence was found to link distal border fragmentation with the degree of lameness. Interestingly, significant association between the presence of distal border fragmentation and lameness was detected only in asymmetrically affected animals. However, in another research, an association was found between the fragments and the overall navicular bone grade in radiological evaluation. Fragments were also strongly associated with primary navicular pathology (Biggi and Dy s on 2012). The same conclusion about the presence of fragments and the overall navicular bone grade was reached in a magnetic resonance imaging investigation. Additionally, this study reported the accordance between distal border fragments and other pathological MRI (Magnetic Resonance Imaging) findings in the navicular bone (Biggi and Dy son 2011). In a case report by Biggi et al. (2012), a large fragment embedded in the DSIL at the junction between distal horizontal and lateral sloping borders was found to be one of the main causes of pain and lameness in a horse. Nonetheless, in a recent MRI examination, conclusions were completely different. There was no evidence to link the presence of a distal border fragment with the lameness. There also was no difference in the size of fragments across the severity grades of lameness. Surprisingly, the average grade of lameness was slightly lower for limbs with fragments, but this finding was not significant (Yorke et al. 2014).

Fragments appear in both lame and non-lame horses. In the study of Biggi and Dyson (2012), fragments were observed in $3.6 \%$ of sound and $8.7 \%$ of lame horses, with majority of them in the lame limb. In another research from the same authors, fragments occurred in $13.6 \%$ of horses with foot pain, with $75 \%$ of them in the lame limb (Biggi and Dyson 2011). Such differences in results from the same authors may arise from different number of horses in each study and different diagnostic techniques. In other researchers' studies, fragments were seen in $28.8 \%$ of lame horses (Wright 1993) and in 7\% of sound horses 
(Kaser-Hotz and Ueltschi 1992). Discrepancies in data may be caused by different sizes of experimental groups, individual features, breeds, and grade of lameness.

Some studies undeniably link fragments with the distal margin of the flexor surface of the bone (Wright 1993; Biggi and Dyson 2011; Biggi and Dyson 2012; Zani et al. 2016). The association between the size of distal extension of the palmar cortex and the occurrence of a distal border fragment was found in MRI studies (Biggi and Dyson 2011; Zani et al. 2016). In the radiological study by Biggi and Dyson, most fragments were associated with an irregular margin of the distal border, but no significant association with the size or thickening of the distal extension of the palmar cortex was found (Biggi and Dyson 2012). Seemingly, the extension of the distal margin could be indeed a factor causing fraction, but it also has been proposed that elongation of the palmar cortex may be a normal variation without being particularly connected with pathological changes. In one radiological investigation of sound horses, elongation of the distal border was found in 55\% of them (Kaser-Hotz and Ueltschi 1992). A supporting argument can also be found in a research investigating the age-related changes in the anatomical shape and histological architecture of healthy navicular bones. In this study, an increase in the distal border volume with age was observed (Gabriel et al. 1999). It may be explained by the process of development of the navicular bone (Rijkenhuizen et al. 1989b).

Taking all of this into consideration, the phenomenon of distal border fragments remains still unclear. They may differ in places and ways of origin and vary in consequences of their occurrence. As a certainty, a close relation between fragments and the distal margin of the navicular bone may be considered, as well as their association with some other lesions of equine digit structures. Some data show that the future carrier of sport horses with distal border fragments is statistically not different from horses without these fragments (Ecrement 2011). Although clinical significance of distal border fragments is controversial, they should not be underestimated when considering the causes of equine lameness. Adaptations in micro- and macro-architecture of the navicular bone in response to stress are to some degree a natural reaction, but when turnover of the bone exceeds the indeterminate threshold, they may became pathological.

\section{Synovial invaginations and other changes within nutrient foramina}

On the distal aspect of the navicular bone, below a small articular surface for the coffin bone, there is a recess formed by synovia called the synovial groove, with nutrient foramina leading to the interior of the bone. According to data from Poulos and Smith (1988), channels in the navicular bone are not lined with blood vessels or vascular endothelium, but represent invaginations of synovial tissue from the synovial fossae with blood vessels associated with it. There are several shapes of the synovial invaginations that can be distinguished as: 'conical', 'linear', 'lollipop' or 'branched', and they can differ also in the depth of penetration (Claerhoudt et al. 2011). Inside the synovial invaginations, there are nerve fibres located entering through them to the navicular bone (Rijkenhuizen et al. 1989c). Plenty of nerve fibres associated with the vasculature are also found in the CSLs and DSIL (Bowker et al. 1994). The presence of these fibres is crucial when considering pain during lameness.

When considering possible causes of pathological nutrient foramina, it is important to understand the significance of the navicular bone's distal border in blood supply. The navicular bone in the adult horse has two principal routes of blood supply, the proximal and the distal ones. In the foetus, the distal source is derived from the network of arteries lying in the DSIL. Immediately after birth, the blood supply undergoes changes in response to the demands of increasing function and work load, and the distal border of the bone is the place of the most intense modifications. Branches from distal arteries gradually penetrate 
the bone, and form into cones of anastomosing vessels responsible for the development of the large foramina along the distal border (Colles and Hickman 1977). In a healthy adult horse, there is a uniform specific pattern characterized by 4 to 8 arteries entering distally, covering the central and distal part of the navicular bone, and 9 to 14 proximal arteries covering the proximal part of the bone. The medial and lateral parts receive supply from the arteries entering medially and laterally (Rijkenhuizen et al. 1989a). Abnormalities in this pattern can lead to insufficient nutrition of particular sections of the navicular bone, and can be a stimulus to other changes in equine digit.

In some cases, normal nutrient foramens can undergo changes and became pathological (Plate III, Fig. 4). The most frequently mentioned signs of nutrient foramina pathology are changes in their size, shape, and number. In a study by Claerdhoudt et al. (2011), an average of 5.9 invaginations per the navicular bone were found. Navicular bones with a total number of 1 to 7 distal border synovial invaginations on radiographs are considered normal, and a higher number can be a sign of pathology. A few other studies suggest that normal nutrient foramina are conical, and should be considered abnormal only in the case of an increased number of them or changes in shape, especially for flask or lollipop shape (Colles and Hickman 1977; MacGregor 1984; MacGregor 1986). However, the conically shaped foramina can also be associated with the navicular disease, when they occur in the wings of the navicular bone (MacGregor 1984). This leads to the conclusion that the location of the foramina may also be important in the considerations of their significance.

There are a few theories about the origin of pathological nutrient foramina modifications. One of them indicates circulatory disturbances (Colles and Hickman 1977; Rijkenhuizen et al. 1989c). In navicular disease, occlusion of the distal vessels may occur due to thrombus formation within the vessel lumen. This phenomenon leads to the development of compensation supply and increased vascularization around nutrient foramina. Following these changes, osteoporosis may develop at their tip, making it rounded or mushroomshaped. Ischaemic changes in the navicular bone may be a serious cause of pain and lameness in the navicular disease (Colles and Hickman 1977). This theory is supported by other observations, where the presence of radiologically visible nutrient foramina was associated with a changed pattern in the arteriogram, fibrosis, and increased bone remodelling in which bone resorption predominated. Ischaemia and increased pressure were considered to be responsible for modifications in the form and number of nutrient foramina (Rijkenhuizen et al. 1989c). The data about active remodelling due to abnormal and dilated synovial invaginations are presented in a study by Ostblom et al. (1982), in which altered pressure from the deep digital flexor tendon on the bone and increased load on the caudal part of the foot are pointed out as the primary source of nutrient foramina changes. Colles and Hickman (1997) also suggest that the size of synovial invaginations does not appear to be related to age, but rather to the type, regularity, and frequency of work performed.

As in case of the distal border fragments, the phenomenon of pathological nutrient foramina should be analyzed in the context of the whole anatomical construction of the equine digit. Abnormal synovial invaginations may be associated with other navicular bone abnormalities. Significant association between the presence of distal border fragments and the size and number of the synovial invaginations was found in studies by Biggi and Dyson (Biggi and Dyson 2011; Biggi and Dyson 2012).

It is also worth noting that pathological nutrient foramina do not have to be linked to the navicular disease. There is also another approach represented by Kaser-Hotz and Ueltschi (1992) suggesting that although there is no question that horses with navicular disease have a higher incidence of abnormal invaginations, they may not be conclusive for the diagnosis of navicular disease. Pathological foramina can be a sign of arthrosis of the 
distal interphalangeal joint and not primarily a result of navicular disease. Observations confirming this theory were revealed in a recent study by Olive and Videau (2017), in which direct communication between the distal border synovial invaginations and the distal interphalangeal joint space was discovered, but connection between the invaginations and the navicular bursa was not observed. Therefore, extended nutrient foramina are likely to be a symptom of distal interphalangeal joint synovitis or osteoarthritis.

Although enlargement of the nutrient foramina is well known and documented in a variety of sources, the obliteration and even complete disappearance of the foramina can be also observed, probably due to a course of bone-tissue turnover (Komosa et al. $2013 b$ ). However, the phenomenon of the obliteration of nutrient foramina requires further, insightful analysis especially in the histological context. In the macroscopic view, the obliteration may occur in a varied form and often the effacement of the synovial groove can be seen (Plate III, Figs 5, 6).

\section{Breed and morphotype}

Studies of navicular bone's abnormalities are undertaken more frequently in breeds of warmblood horses than in coldblood ones. This is due to a high popularity of horseback riding and a lower interest in the use of harness horses. Because of this disparity in the quantity of studies, a problem of the navicular bone's changes is better known in warmblood horses, although there are still a lot of unexplained issues. The variety of forms of the disease's changes impede the exploration of this topic. Often a few pathological changes occur together, like in the instance of the origin of the synovial groove and distal border fragments. Intensive use of sport horses and requirements on equestrian events are in particular linked to a heavy load on the forelegs. The accumulation of small injuries during gallop and jumping can be the key cause of pathological changes of the distal sesamoid bone. The type of ground most often used in training of the horse is also important (Komosa et al. 2014).

In light of the data explained above, the condition of navicular bones of coldblood horses, in which comprehensive radiological and anatomical examinations are rare, is striking. This category of horses, due to a different purpose of use, are not subjected to the same type of load as saddle horses. Furthermore, they are characterized by a specific morphotype expressed in their unique skeleton features. Unlike their warmblood counterparts, coldblood horses have massive bones, in many cases also different in shape. Different width to length proportions were found especially in the analysis of the autopodial segments of limbs (Alho 1993; Dzierzęcka and Komosa 2013). Also the bone's architecture, understood by the ratio of the compact bone to the spongy substance, differs in horses of various morphotypes (Firth et al. 2005; Dzierzęcka and Charuta 2012). Some data on the anatomy of the distal sesamoid bone of various breeds of horses were reported by studies of Gabriel et al. (1998), however, this topic still remains open to further investigations. Conclusions of the above mentioned authors suggest that navicular bones can vary in the spatial structure and react differently to load, according to the type or breed of horses. For this reasons, we suppose that exploring variations of the navicular bone's changes in warmbloods and coldbloods may contribute to a better understanding of the problem of this bone's pathology (Hickman 1989).

There is some evidence that the navicular disease may be linked to genetic predispositions, pointing out that warmblood horses are more susceptible (Diesterbeck and Distl 2007; Finne 2014). Quarter horses and thoroughbreds seem to be prone to navicular bone changes to a higher degree than other breeds (Lowe 1976). In a study of 3748 young Hanoverian warmblood horses by Stock et al. (2004) a higher percentage of genes of the Hanoverian and the Holstein warmblood horses was found as a factor increasing the 
probability of occurrence of some pathological changes within the navicular bone. Results of older studies showed that podotrochleosis associated with the pathology of the navicular bone may depend partly on the presence of genetic factors (Bos et al. 1986).

In the majority of the discussed academic works there is no information on the association between the incidence of navicular pathology and the breed and discipline of the horses. The disease was not found to be related to the breed, sex or use of the horses in one of the older studies (Ostblom et al. 1982). There was also no association between the presence of the distal border fragment and the height, weight, breed, age or discipline of the horses in the study by Biggi and Dyson (2011).

A study by Yorke et al. (2014) in which there was no increased probability of being categorized as lame if a fragment was present, distal border fragments were most frequent in warmblood horses $(36.7 \%)$. Other breeds were represented in the following proportions: Quarter horses (26.7\%), Arabians (10\%), Thoroughbreds (8.3\%), Appaloosa (1.7\%), Irish Sport horse (1.7\%), Paint (1.7\%), Percheron (1.7\%), and mixed breed or unknown $(11.7 \%)$. Group percentages were comparable to the overall hospital caseload; despite different numbers mentioned above, no significant association between distal border fragments and the breed was found. There was also no relation between fragments and the sex or age of the animal.

Reliable information in the literature regarding the importance of breed in the pathomorphology of the distal border of the navicular bone is scarce. The majority of studies are focused on the process of the origin of lameness, associations between lesions, and the clinical importance of this changes. The breed seems to be a factor related to navicular bone pathology, but authors work with a variety of breeds and this issue is not always taken into consideration. It is also not easy to separate the influence resulting from innate qualities of different breeds and the reasons derived from different purposes of these groups of horses and the different categories of load they are subjected to.

In conclusion, the navicular bone, despite its small size, has a big impact on the health and condition of the horse. This structure is crucial for proper distribution of the burden during locomotion, and because of this the function is subjected to mechanical stress caused by neighboring elements. Response to load is the turnover of bone tissue, resulting in changes of the architecture of the navicular bone. Such adaptation is a natural phenomenon, but if too intense, it can cause pathological changes in the bone and progressive lameness of the horse. The majority of alterations can be found on the distal margin of the bone, which is the surface experiencing a serious load during movement, and playing the main role in blood supply of the whole structure. Such changes as enthesophytes and distal border fragments can injure other elements of the equine limb and be the cause of severe pain. Obviously, such findings vary in individual cases. They differ in the origin, site, size, and form. For this reason, each incident should be considered separately. The presence of osseous alterations do not need to be associated with a serious condition, however, they should never be underestimated, particularly if they are large in size or occurring with other lesions. A similar position should be taken against alterations of the nutrient foramina. Uneven and inadequate blood supply to the navicular bone associated with them can have fateful consequences. The degree of such changes should always be considered. Various lesions can coexist in many combinations, and consequently be a sign of a more advanced pathological condition. At the same time, concurrent alterations may impede the recognition of the main original cause of the navicular disease. The influence of genetic predispositions and type of stress affecting different categories of horses is still not well known. To better understand the above described problems and to evaluate the condition of horses more effectively, further studies are needed. They should cover extensive experimental groups of horses of diverse breeds, take into account the medical and sport history of individual horses, and apply several types of diagnostic methods. 


\section{References}

Alho A 1993: Mineral and mechanics of bone fragility fractures. Acta Orthop 64: 227-232

Bentley VA, Sample SJ, Livesey MA, Scollay MC, Radtke CL, Frank JD, Kalscheur VL, Muir P 2007: Morphologic changes associated with functional adaptation of the navicular bone of horses. J Anat 211: 662-672

Biggi M, Blunden T, Dyson S 2012: Can distal border fragments of the navicular bone be a primary cause of lameness? Equine Vet Educ 25: 347-351

Biggi M, Dyson S 2010: Comparison between radiological and magnetic resonance imaging lesions in the distal border of the navicular bone with particular reference to distal border fragments and osseous cyst-like lesions. Equine Vet J 42: 707-712

Biggi M, Dyson S 2011: High-field magnetic resonance imaging investigation of distal border fragments of the navicular bone in horses with foot pain. Equine Vet J 43: 302-308

Biggi M, Dyson S 2012: Distal border fragments and shape of the navicular bone: radiological evaluation in lame horses and horses free from lameness. Equine Vet J 44: 325-31

Blunden A, Dyson S, Murray R, Schramme M 2006: Histopathology in horses with chronic palmar foot pain and age-matched controls. Part 1: Navicular bone and related structures. Equine Vet J 38: 15-22

Bos H, van der Meij GJ, Dik KJ 1986: Heredity of the navicular disease. Vet Q 8: 68-72

Bowker RM, Rockershouser SJ, Linder K, Kelly BV, Ioana MS, Caron JP 1994: A silver-impregnation and immunocytochemical study of innervation of the distal sesamoid bone and its suspensory ligaments in the horse. Equine Vet J 26: 212-219

Claerhoudt S, Bergman EHJ, van der Veen H, Vanderperren K, Raes EV, Saunders JH 2011: Computed tomographic morphology of the synovial invaginations of the distal sesamoid bone of the horse. Anat Histol Embryol 40: 55-60

Claerhoudt S, Bergman HJ, van der Veen H, Duchateau L, Raes EV, Vanderperren K, Saunders JH 2011: Morphology of distal border synovial invaginations of the equine distal sesamoid bone: comparison between computed tomography and a hoof-specific radiographic projection. Vet Comp Orthop Traumatol 25: 453-459

Colles CM, Hickman J 1977: The arterial supply of the navicular bone and its variations in navicular disease. Equine Vet J 9: 150-154

Denoix, J-M 1999: Functional anatomy of the equine interphalangeal joints. Proc AAEP 45

Diesterbeck U, Distl O 2007: Review of genetic aspects of radiological alterations in the navicular bone of the horse. Dtsch Tierarztl Wochenschr 114: 404-411

Dyson S 2008: Radiological interpretation of the navicular bone. Equine Vet J 20: 268-280

Dyson S, Murray R, Blunden T, Schramme M 2006: Current concepts of navicular disease. Equine Vet J 18: $45-56$

Dzierzęcka M, Charuta A 2012: The analysis of densitometric and geometric parameters of bilateral proximal phalanges in horses with the use of peripheral quantitative computed tomography. Acta Vet Scand 54: 41-51

Dzierzęcka M, Komosa M 2013: Variability of the proximal phalanx in warmblood and coldblood horses morphological and structural analyses. Belg J Zool 143: 119-130

Ecrement O 2011: Clinical and sport impact of distal fragments of distal sesamoid bone of sport horses. $\mathrm{PhD}$ Thesis University of Alfort pp 1-67

Finne R 2014: Genetics and markers of navicular disease in warmblood sport horses. Available at: http://www. huveta.hu/handle/10832/1283

Firth EC, Rogers CW, Double M, Jopson NB 2005: Musculoskeletal responses of 2-year old Thoroughbred horses to early training. Bone parameters in the third metacarpal and third metatarsal bones. N Z Vet J 53: 101-112

Gabriel A, Detilleux J, Jolly S, Reginster J-Y, Collin B, Dessy-Doizé C 1999: Morphometric Study of the Equine Navicular Bone: Age-Related Changes and Influence of Exercise. Vet Res Commun 23: 15-40

Gabriel A, Jolly S, Detilleux J, Dessy-Doize C, Collin B, Reginster JY 1998: Morphometric study of the navicular bone: variations with breeds and types of horse and influence of exercise. J Anat 193: 535-549

Hickman J 1989: Navicular disease--what are we talking about? Equine Vet J 21: 395-398

Kaser-Hotz B, Ueltschi G 1992: Radiographic appearance of the navicular bone in sound horses. Vet Radiol Ultrasound 33: 9-17

Komosa M, Łazowski S, Włodarek J, Kowalczyk K, Charuta A, Zdun M 2014: Gross and histological evaluation of early lesions of navicular bone and deep digital flexor tendon in horses. Bull Vet Instit in Pulawy 58: 87-91

Komosa M, Purzyc H, Frąckowiak H 2013a: Changes in navicular bone (os sesamoideum distale) shape in horses as a result of pathological alterations. Folia Biol (Krakow) 61: 1-10

Komosa M, Purzyc H, Wojnar M, Frąckowiak H, Kobryńczuk F 2013b: Navicular syndrome in sport horses as a result of the disorder of biological bone tissue turnover rhythm: A review. Biol Rhythm Res 44: 339-351

Lowe JE 1976: Sex, breed, and age incidence of navicular disease. Proc AAEP 37

MacGregor CM 1984: Studies on the pathology and treatment of equine navicular disease. PhD thesis, University of Edinburgh

MacGregor CM 1986: Radiographic assessment of navicular bones, based on changes in the distal nutrient foramina. Equine Vet J 18: 203-206

Olive J, Videau M 2017: Distal border synovial invaginations of the equine distal sesamoid bone communicate with the distal interphalangeal joint. Vet Comp Orthop Traumatol 2: 107-110 
Ostblom L, Lund C, Melsen F 1982: Histological study of navicular bone disease. Equine Vet J 14: 199-202

Poulos PW, Smith MF 1988: The nature of enlarged 'vascular channels' in the navicular bone of the horse. Vet Radiol Ultrasound 29: 60-64

Rijkenhuizen AB, Nemeth F, Dik KJ, Goedegebuure SA 1989a: The arterial supply of the navicular bone in the normal horse. Equine Vet J 21: 399-404

Rijkenhuizen AB, Németh F, Dik KJ, Goedegebuure SA 1989b: Development of the navicular bone in foetal and young horses, including the arterial supply. Equine Vet J 21: 405-412

Rijkenhuizen AB, Németh F, Dik KJ, Goedegebuure SA 1989c: The arterial supply of the navicular bone in adult horses with navicular disease. Equine Vet J 21: 418-424

Sandler EA, Kawcak CE, McIlwraith CW, Norrdin RW 2000: Remodeling of the navicular bone in response to exercise - a controlled study. AAEP Proc 46: 46-50

Stock KF, Hamann H, Distl O 2004: Variance component estimation on the frequency of pathologic changes in the navicular bones of Hanoverian Warmblood horses. J Anim Breeding Gent 121: 289-301

Waguespack RW, Hanson RR 2010: Navicular syndrome in equine patients anatomy, causes, and diagnosis. Compend Contin Educ Vet 32: 1-13

Wilson A, Weller R 2011: Diagnosis and Management of Lameness in the Horse, chapter 26: The Biomechanics of the Equine Limb and Its Effect on Lameness. Elsevier Saunders 270-281

Wilson AM, McGuigan MP, Fouracre L, MacMahon L 2001: The force and contact stress on the navicular bone during trot locomotion in sound horses and horses with navicular disease. Equine Vet J 33: 159-165

Wright IM 1993: A study of 118 cases of navicular disease: radiological features. Equine Vet J 25: 493-500

Wright IM, Kidd L, Thorp BH 1998: Gross, histological and histomorphometric features of the navicular bone and related structures in the horse. Equine Vet J 30: 220-34

Yorke EH, Judy CE, Saveraid TC, McGowan CP, Caldwell FJ 2014: Distal border fragments of the equine navicular bone: association between magnetic resonance imaging characteristics and clinical lameness. Vet Radiol Ultrasound 55: 35-44

Zani de D, Polidori C, Giancamillo di M, Zani DD 2016: Correlation of radiographic measurements of structures of the equine foot with lesions detected on magnetic resonance imaging. Equine Vet J 48: 165-171 
Plate II

Frątczak M. et al.: Insight into ... pp. 123-131

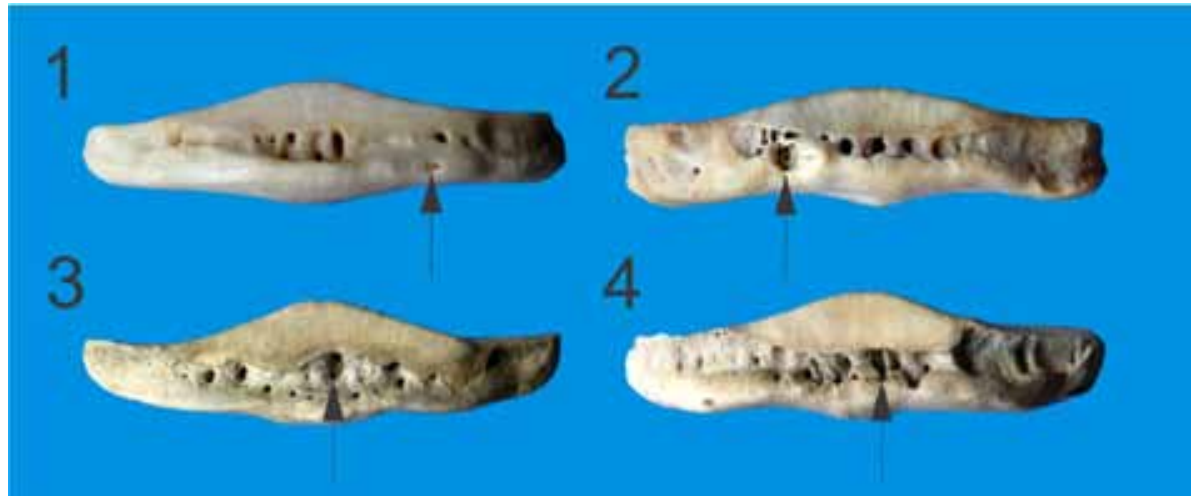

Fig. 1. The variety of the distal border's pathological alterations. No. $1-$ the arrow indicates the place after the fraction of a small osseous fragment, adjacent to disappearing nutrient foramina. No. 2 - the place after big-sized fragment accompanying enlarged nutrient foramina. No. 3 - the enlarged synovial groove and reduction of the articular surface for a contact with the coffin bone. No. 4 - strong synovial invaginations in the central part of the bone.

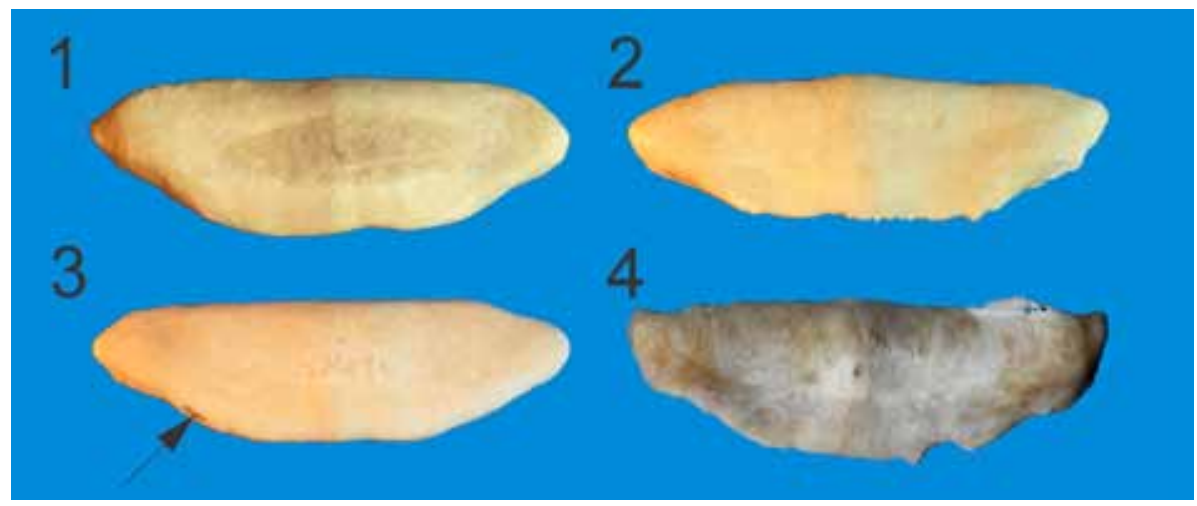

Fig. 2. Alterations visible on the flexor surface. No. 1 - the navicular bone's asymmetry resulting from uneven load and bone's superstructure on one side. No. 2 - the rugged distal border of the bone, visible minor new bone formations. No. 3 - the small splinter of the bone on the lateral part of the distal margin, indicated by the arrow. No. 4 - the large fracture of bone fragment and a sharpened margin in the place of loss.

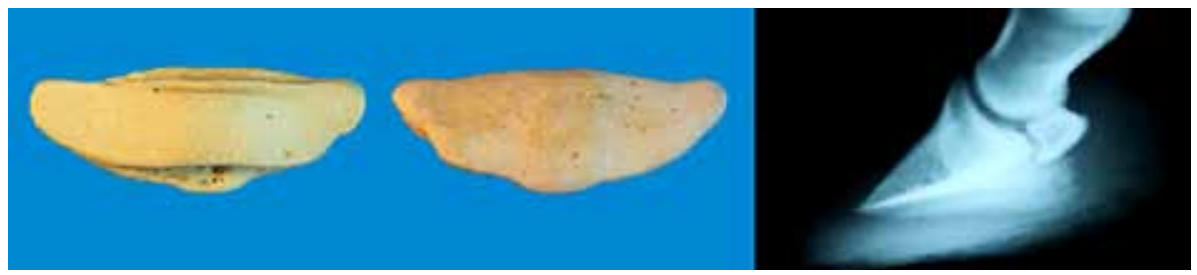

Fig. 3. Strongly arched central part of the distal border. The view of articular surface to the left and flexor surface to the right. The same navicular bone on the radiological image in lateral projection. Also thickened cortical layer of the bone can be seen. 


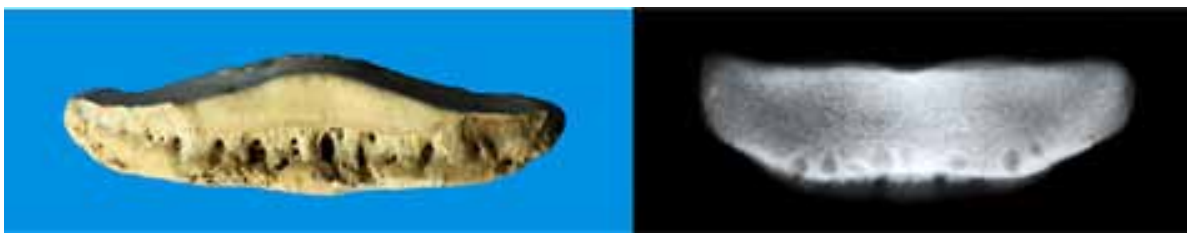

Fig. 4. Comparison between anatomical and radiological images of synovial invaginations. Mildly and moderately penetrating, lollipop-shaped canals can be well seen.

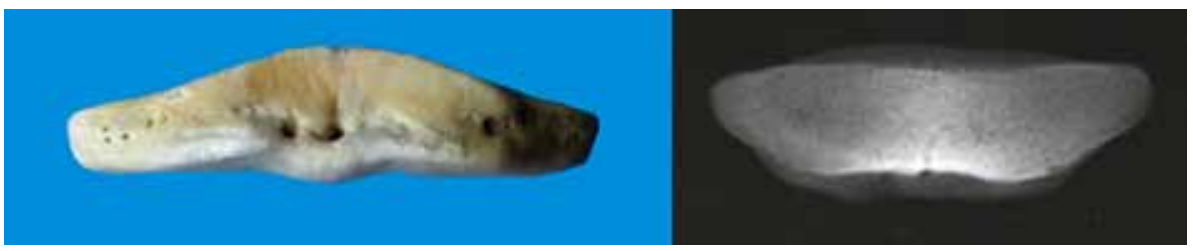

Fig. 5. The vanishing of the synovial groove and obliteration of the majority of nutrient foramina. The radiographic image shows preserved synovial invaginations, in which we can derive two linear and one conical canals. Only one canal deeply penetrates the bone, others underwent a process of atrophy together with foramina.

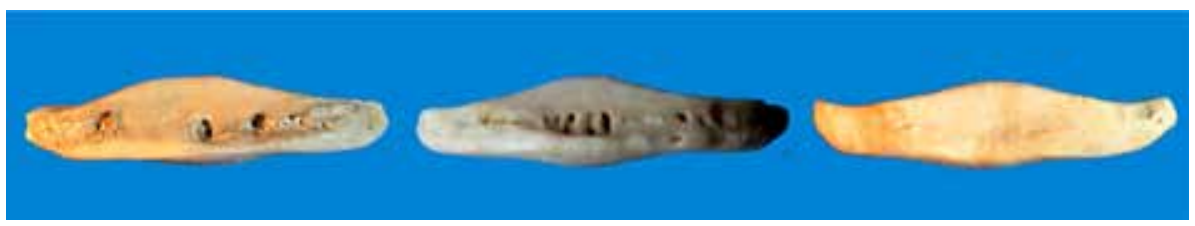

Fig. 6. Distal borders of the navicular bones with nutrient foramina obliterated to varying degrees. Far right, view of the complete disappearance of the foramina and synovial groove. 